\title{
A COUPLED VEM-BEM APPROACH FOR COMPUTATIONAL HOMOGENIZATION OF HETEROGENEOUS MATERIALS
}

\author{
M. LO CASCIO ${ }^{1, a}$, A. MILAZZO ${ }^{1, b}$, I. BENEDETTI ${ }^{1, c}$ \\ ${ }^{1}$ Department of Engineering, University of Palermo \\ Viale delle Scienze, Edificio 8, 90128, Palermo, Italy \\ a marco.locascio01@unipa.it, ${ }^{b}$ alberto.milazzo@unipa.it, ${ }^{c}$ ivano.benedetti@unipa.it
}

Key words: Virtual Element Method, Boundary Element Method, Coupled formulation, Computational Micromechanics, Composite materials.

\begin{abstract}
The Boundary Element Method (BEM) is well established as an accurate and powerful numerical technique in continuum mechanics. Due to its intrinsic feature of reducing the problem's dimensionality, which allows reducing the modelling effort without compromising on the solution accuracy, the BEM has been successfully employed for the computational homogenization of materials with complex morphologies. The Virtual Element Method (VEM) has recently emerged as a powerful and robust technique, capable of handling very general polygonal/polyhedral mesh elements. Such a property is of interest in treating problems whose analysis domain presents complex geometric features, as it simplifies the data preparation stage of the analysis. In this work, we use a coupled VEM-BEM approach for computational homogenization of heterogeneous materials whose microstructure is characterized by inclusions of irregular shapes embedded in a surrounding matrix.
\end{abstract}

\section{INTRODUCTION}

The Finite Element Method (FEM) [1] and the Boundary Element Method (BEM) [2] are numerical techniques widely employed for the solution of several classes of problems in solids and materials mechanics. FEM allows generality and relative simplicity in including complex modelling aspects, such as domain inhomogeneity or non-linear material behaviours. BEM is known to offer pre-processing simplification and high numerical accuracy at a relatively reduced computational cost, especially in problems requiring accurate representation of surfaces. For some classes of problems exhibiting regions with different properties, acknowledging such benefits has suggested coupling the two methods in hybrid approaches combining their relative strengths $[3,4,5,6]$.

The Virtual Element Method (VEM) [7] extends the features of FEM to very general polygonal and polyhedral mesh elements, including irregular or non-convex ones. For such a reason, VEM provides appreciable simplification in the data preparation stage of the analysis, especially in problems whose analysis domain features complex geometries, e.g., computational micro-mechanics problems [8, 9, 10, 11, 12], which may induce irregular meshes when automatic algorithms are used. BEM, on the other hand, has also been successfully employed for the computational homogenisation of materials with complex morphology or constitutive behaviour [13, 14, 15, 16].

In this contribution, we explore the capabilities of a coupled VEM-BEM numerical technique [17] for 


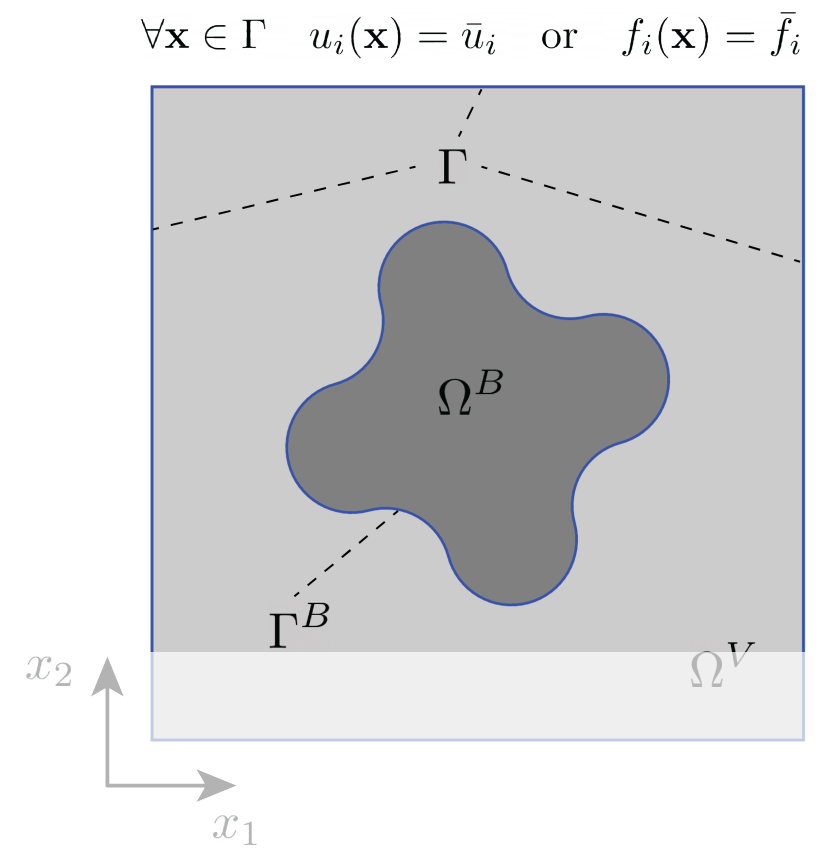

Figure 1: Reference model for the coupled VEM/BEM approach.

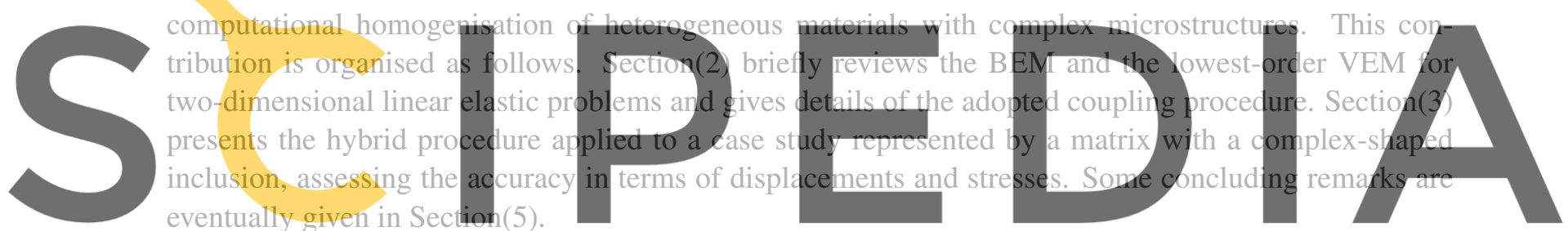

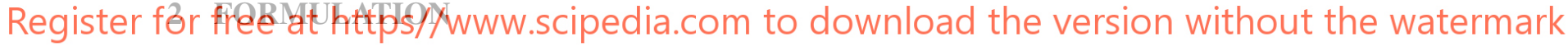

Let us consider a two-dimensional linear elastic problem set in the framework of small strains. The

elastic body lies within the domain $\Omega \in \mathbb{R}^{2}$ with its external boundary $\Gamma$. It is assumed that no body forces act within $\Omega$, but both tractions and/or displacements can be enforced on the boundary $\Gamma$. The domain $\Omega$ is considered as the union of two subdomains, namely $\Omega^{\mathrm{B}}$ and $\Omega^{\mathrm{V}}$ that represent, respectively, an irregularly shaped inclusion, representative e.g. of the transversal section of a fibre in a polymer fibre-reinforced composite, and the surrounding matrix, representative e.g. of the polymer matrix in the mentioned class of materials. The two subdomains share the interface $S$, as shown in Fig.(1), where the considered analysis domain is drawn with its features.

In the proposed approach, BEM is used to model the inclusion while VEM is used to model the surrounding matrix. To this end, the domain $\Omega^{\mathrm{V}}$ is partitioned into a number of polygons of general shape, while the boundary $S$ is divided into a number of segments, which form the edges of the polygonal elements in $\Omega^{\mathrm{V}}$ lying in proximity of the interface between the two subdomains. 


\subsection{BEM formulation}

For the BEM subdomain $\Omega^{\mathrm{B}}$ with boundary $S$, in absence of body forces and using tensor notation with $i, j=x, y$, the boundary integral equation (BIE) for the displacements $u_{j}$ at a boundary collocation point $\mathbf{x}_{0} \in S$ can be written as [2]

$$
\alpha_{i j}\left(\mathbf{x}_{0}\right) u_{j}\left(\mathbf{x}_{0}\right)=\int_{S} G_{i j}\left(\mathbf{x}_{0}, \mathbf{x}\right) t_{j}(\mathbf{x}) d s-\int_{S} H_{i j}\left(\mathbf{x}_{0}, \mathbf{x}\right) u_{j}(\mathbf{x}) d s
$$

where the terms $\alpha_{i j}$ are functions of the geometry at the point $\mathbf{x}_{0}, u_{i}(\mathbf{x})$ and $t_{i}(\mathbf{x})$ are unknown displacement and traction components at the integration boundary point $\mathbf{x}$. The fundamental solutions $H_{i j}\left(\mathbf{x}_{0}, \mathbf{x}\right)$ and $G_{i j}\left(\mathbf{x}_{0}, \mathbf{x}\right)$ for plane strain condition are given by

$$
\begin{gathered}
G_{i j}\left(\mathbf{x}_{0}, \mathbf{x}\right)=C_{1}\left(C_{2} \delta_{i j} \ln r-r_{, i} r_{, j}\right) \\
H_{i j}\left(\mathbf{x}_{0}, \mathbf{x}\right)=\frac{C_{3}}{r}\left[n_{k} r_{, k}\left(C_{4} \delta_{i j}+2 r_{, i} r_{, j}\right)-C_{4}\left(r_{, i} n_{j}-r_{, j} n_{i}\right)\right]
\end{gathered}
$$

where $r=\left|\mathbf{x}-\mathbf{x}_{0}\right|$ is the Euclidean distance between the points $\mathbf{x}_{0}$ and $\mathbf{x}$, the indicial notation $f_{, i}=\partial f / \partial x_{i}$ is adopted to refer to differentiation, $n_{i}$ are components of the outward unit normal vector to the boundary $S$ at the generic smooth point $\mathbf{x}$. The coefficients $C_{1}, C_{2}, C_{3}$ and $C_{4}$ are given by

$$
C_{1}=-\frac{1+v}{4 \pi(1-v) E}, \quad C_{2}=3-4 v, \quad C_{3}=-\frac{1}{4 \pi(1-v)}, \quad C_{4}=1-2 v,
$$

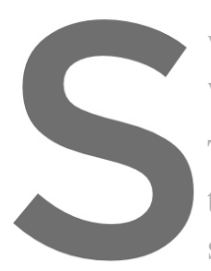

with $E$ and $v$ denoting respective $y$ the Young's modulus and the Poisson's ratiog the isotropic material
within the subdomain $S^{B}$.
The numerical solution of Eq.(1) is based on the discretisation of $S$ and the successive approximation of
the boundary displacement and traction components in terms of shape functions and nodal values More
specifically, $S$ is subdivider into $m$ straight segment $\Delta S_{k}$, and two nodes are associated with the extremes

of each segment; each node carries two components of displacements and two components of tractions.

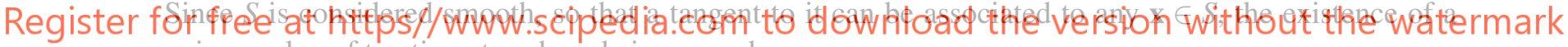
unique value of traction at each node is ensured.

Both displacement and traction components are assumed to be globally continuous over $S$ and to vary linearly over each boundary segment $\Delta S_{k}$ according to

$$
\mathbf{u}(\xi)=\mathbf{N}(\xi) \mathbf{u}^{k}, \quad \mathbf{t}(\xi)=\mathbf{N}(\xi) \mathbf{t}^{k}
$$

where $\mathbf{N}(\xi) \in \mathbb{R}^{2 \times 4}$ is the matrix collecting 1D linear shape functions for the boundary segment $\Delta S_{k}$, expressed as function of the natural coordinate $\xi$, and $\mathbf{u}^{k}, \mathbf{t}^{k} \in \mathbb{R}^{4 \times 1}$ collect, respectively, nodal components of displacements and tractions associated with the boundary segment $\Delta S_{k}$.

Eq.(1), collocated at the generic boundary node $p$, may be rewritten in matrix notation as

$$
\alpha \mathbf{u}_{p}=\sum_{q=1}^{m}\left[\int_{\Delta S_{q}} \mathbf{G}_{p q}(\xi) \mathbf{N}(\xi) J(\xi) d \xi\right] \mathbf{t}^{q}-\sum_{q=1}^{m}\left[\int_{\Delta S_{q}} \mathbf{H}_{p q}(\xi) \mathbf{N}(\xi) J(\xi) d \xi\right] \mathbf{u}^{q}
$$

where $\alpha \in \mathbb{R}^{2 \times 2}$ is a matrix depending on the geometry of the boundary at the considered collocation point $p$, smooth in this case, $\mathbf{u}_{p} \in \mathbb{R}^{2 \times 1}$ collects the components of displacements at the node $p, \mathbf{u}^{q}$ and $\mathbf{t}^{q}$ 
are nodal displacements and tractions associated with $\Delta S_{q}$, as in Eq.(4), and $J(\xi)$ indicates the absolute value of the Jacobian of the transformation between segment local and natural coordinates.

After integration and appropriate algebraic manipulations, we can rewrite Eq.(5) in compact form as

$$
\mathbf{H}_{p} \mathbf{U}^{\mathrm{B}}=\mathbf{G}_{p} \mathbf{T}^{\mathrm{B}}
$$

where $\mathbf{H}_{p}, \mathbf{G}_{p} \in \mathbb{R}^{2 \times 2 m}$ denote the rectangular matrices obtained by collocating at the node $p$ and integration over the whole boundary $S$ while $\mathbf{U}^{\mathrm{B}}, \mathbf{T}^{\mathrm{B}} \in \mathbb{R}^{2 m \times 1}$ collect the components of displacements and tractions for all the nodes identified on the boundary $S$, with the superscript B introduced to highlight that such quantities are associated with the BEM domain. Writing Eq.(6) $\forall p \in[1, \ldots, m]$, we obtain the set of linear algebraic equations

$$
\mathbf{H} \mathbf{U}^{\mathrm{B}}=\mathbf{G} \mathbf{T}^{\mathrm{B}}
$$

where $\mathbf{H}, \mathbf{G} \in \mathbb{R}^{2 m \times 2 m}$ collect the coefficient obtained from the integration of Eq.(5) written for all the collocation points. Since the BEM subdomain identifies an inclusion in the analysed domain, both $\mathbb{U}^{\mathrm{B}}$ and $\mathbf{T}^{\mathrm{B}}$ are unknown quantities that must be determined by interfacing Eq.(7) with the equations obtained by the numerical model of the complementary matrix domain.

\subsection{VEM formulation}

We use the lowest-order VEM for linear two-dimensional elastostatics problems to model the subdomain $\Omega^{\mathrm{V}}$, which is discretised with general polygonal elements, including highly distorted or non-convex ele-
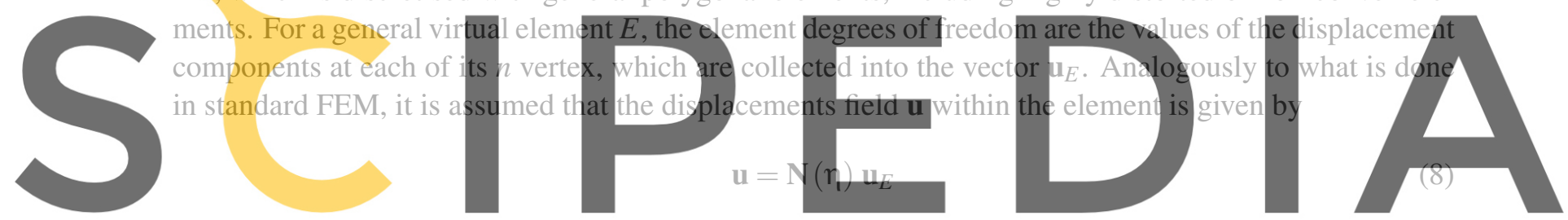

where $\mathbf{N}(\eta)$ is the generic matrix containing the virtual shape functions $N_{v}(\eta)$ associated with each ver-

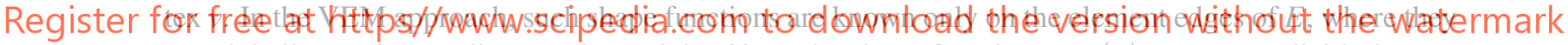
are globally continuous linear polynomials. Since the shape functions, $N_{v}(\eta)$ are not explicitly known within the polygonal element, an explicit expression for the strains is not available. An approximated constant strain field $\varepsilon_{\pi}$ is assumed within each element, which can be computed from the degrees of freedom $\mathbf{u}_{E}$ as

$$
\varepsilon_{\pi}=\Pi_{E} \mathbf{u}_{E}
$$

where $\Pi_{E} \in \mathbb{R}^{3 \times 2 n}$ is the matrix representation of a projection operator, defined as [18]

$$
\Pi_{E}=\frac{1}{A_{E}} \sum_{v=1}^{n} \int_{e_{v}} \mathbf{N}_{v}^{E} \mathbf{N}(\eta) d s
$$

where $A_{E}$ is the area of the polygonal element $E$, bounded by its $n$ edges $e_{v}$ and

$$
\mathbf{N}_{v}^{E}=\left[\begin{array}{cc}
n_{x} & 0 \\
0 & n_{y} \\
n_{y} & n_{x}
\end{array}\right]
$$


is the matrix containing the components $n_{x}$ and $n_{y}$ of the outward unit normal vector over each edge. The integrals appearing at the right-hand side of Eq.(10), which are carried out over the element edges, are exactly computable, since the restriction of the virtual shape functions $N_{v}$ to such edges are piecewise linear polynomials.

The VEM elemental stiffness matrix $\mathbf{K}_{E}$ is the sum of two terms

$$
\mathbf{K}_{E}=\mathbf{K}_{E}^{c}+\mathbf{K}_{E}^{s} .
$$

The first term is given by

$$
\mathbf{K}_{E}^{c}=A_{E} \Pi_{E}^{\mathrm{T}} \mathbf{C} \Pi_{E},
$$

where $\mathbf{C}$ represents the material stiffness tensor in Voigt notation. Since the approximate strains $\varepsilon_{\pi}$ are assumed constant within the element, while the displacements $\mathbf{u}$ are piecewise linear on the element edges, in general, $\varepsilon_{\pi}$ are not compatible with the nodal degrees of freedom $\mathbf{u}_{E}$. The computation of the discrete internal strain energy, using only the approximate constant strains $\varepsilon_{\pi}$, may lead to zero-energy modes not associated with rigid body motion. A stabilisation strategy is employed to ensure the proper rank of the $\mathbf{K}_{E}$ by introducing the stabilisation matrix $\mathbf{K}_{E}^{s}$ which be computed as in Ref.[19].

The equivalent nodal forces $\mathbf{F}_{E}$ are computed as in the standard FEM from specified tractions $\overline{\mathbf{t}}$ over the element boundary $\partial E=\bigcup e_{v}$, i.e.

$$
\mathbb{F}_{E}=\int_{\partial E} \mathbb{N}^{T}(\eta) \bar{t} d s
$$
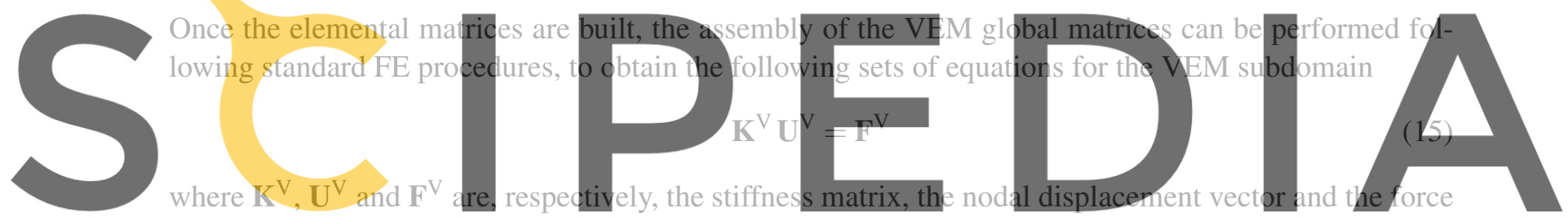

vector for the VEM subdomain, with the superscript $V$ introduced to identify quantities related with the

Register fOFffeemathttps//www.scipedia.com to download the version without the watermark

\subsection{Coupling procedure}

To couple the virtual and the boundary element methods, the BEM subdomain is treated as a macrofinite element, and the traction-displacement equations associated with it are transformed into forcedisplacement equations and assembled with the VEM equations, already expressed in terms of nodal forces and displacements. Finally, equilibrium and compatibility are enforced along the common interface between the VEM subdomain and the BEM subdomain.

The vectors $\mathbf{U}^{\mathrm{V}}$ and $\mathbf{F}^{\mathrm{V}}$ appearing in Eq.(15) collect the displacement and nodal force components of all the VEM nodes in the considered domain. Since only some of such nodes belong to the interface $S$, it is possible to partition the vectors as

$$
\mathbf{U}^{\mathrm{V}}=\left[\begin{array}{c}
\mathbf{U}_{S}^{\mathrm{V}} \\
\mathbf{U}_{D}^{\mathrm{V}}
\end{array}\right], \quad \mathbf{F}^{\mathrm{V}}=\left[\begin{array}{c}
\mathbf{F}_{S}^{\mathrm{V}} \\
\mathbf{F}_{D}^{\mathrm{V}}
\end{array}\right],
$$

where the subscript $S$ and $D$ refer, respectively, to the interface nodes and to the remaining the nodes of the remaining analysis domain. Therefore, we may rewrite Eq.(15) as 


$$
\left[\begin{array}{ll}
\mathbf{K}_{S S} & \mathbf{K}_{S D} \\
\mathbf{K}_{D S} & \mathbf{K}_{D D}
\end{array}\right]\left[\begin{array}{l}
\mathbf{U}_{S}^{\mathrm{V}} \\
\mathbf{U}_{D}^{\mathrm{V}}
\end{array}\right]=\left[\begin{array}{l}
\mathbf{F}_{S}^{\mathrm{V}} \\
\mathbf{F}_{D}^{\mathrm{V}}
\end{array}\right]
$$

Along $S$, the nodal displacements and forces must then satisfy the compatibility and equilibrium conditions

$$
\mathbf{U}^{\mathrm{B}}=\mathbf{U}_{S}^{\mathrm{V}}, \quad \mathbf{F}^{\mathrm{B}}+\mathbf{F}_{S}^{\mathrm{V}}=\mathbf{0},
$$

which have been written considering that no external nodal forces act on the nodes belonging to $S$. The displacement compatibility equations can be readily written, as the same displacement components appear in both the BEM and VEM equations. On the contrary, while nodal forces appear in Eq.(15), related to the VEM domain, tractions appear in Eq.(7), related to the BEM domain, so that it is necessary to retrieve consistent nodal forces from BEM tractions, before writing the equilibrium equations appearing in Eq.(18). This may be done, for a generic boundary element node, by resorting to appropriate energetic considerations. In the adopted scheme, two-node piecewise linear boundary elements are used so that a generic node always lies at the conjunction of two contiguous boundary elements. If the generic node $i$ lies between the boundary elements $\Delta S_{k}$ and $\Delta S_{k+1}$, then, for a virtual displacement $\delta \mathbf{u}\left(\mathbf{x}_{i}\right) \equiv \delta \mathbf{u}_{i}$ of the node $i$, the unknown nodal force $\mathbf{F}_{i}^{\mathrm{B}}$ will perform a work that should be energetically equivalent to the work performed by the tractions acting on the two contiguous boundary elements. Thus, the following equivalence holds
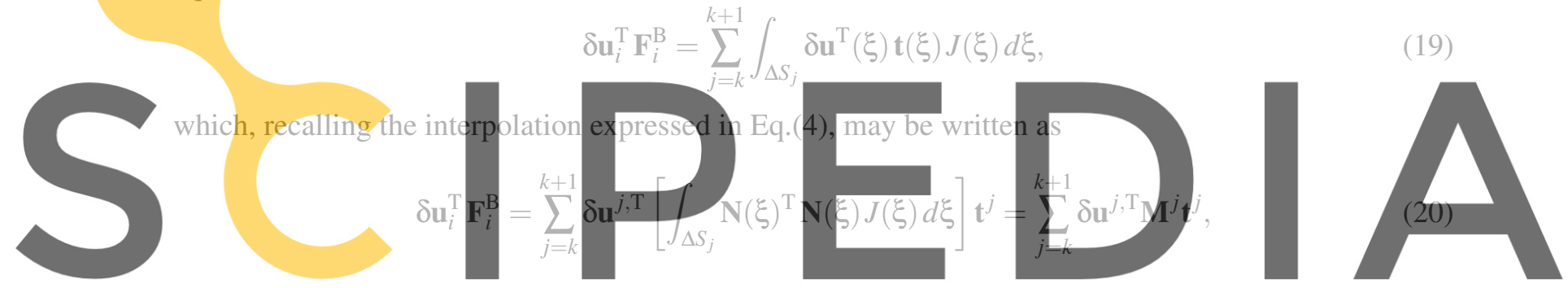

where $\mathbf{M}^{j} \in \mathbb{R}^{4 \times 4}$ stem from the integration over the considered elements of the shape functions matrices,

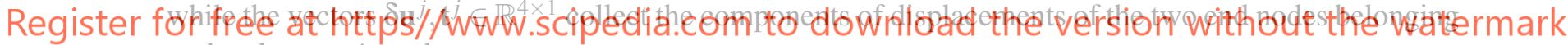

to the element $j$, so that

$$
\delta \mathbf{u}^{k}=\left[\begin{array}{l}
\delta \mathbf{u}_{i-1} \\
\delta \mathbf{u}_{i}
\end{array}\right]=\left[\begin{array}{c}
\mathbf{0} \\
\delta \mathbf{u}_{i}
\end{array}\right], \quad \delta \mathbf{u}^{k+1}=\left[\begin{array}{l}
\delta \mathbf{u}_{i} \\
\delta \mathbf{u}_{i+1}
\end{array}\right]=\left[\begin{array}{c}
\delta \mathbf{u}_{i} \\
\mathbf{0}
\end{array}\right]
$$

Taking into account Eqs.(21), Eq.(20) may be rewritten

$$
\delta \mathbf{u}_{i}^{\mathrm{T}} \mathbf{F}_{i}^{\mathrm{B}}=\delta \mathbf{u}_{i}^{\mathrm{T}} \sum_{j=k}^{k+1} \tilde{\mathbf{M}}^{j} \mathbf{t}^{j} \Rightarrow \mathbf{F}_{i}^{\mathrm{B}}=\sum_{j=k}^{k+1} \tilde{\mathbf{M}}^{j} \mathbf{t}^{j}
$$

where $\tilde{\mathbf{M}}^{j} \in \mathbb{R}^{2 \times 4}$ is the sub-matrix extracted from $\mathbf{M}^{j}$ selecting the appropriate rows corresponding to the displacements associated with the node $i$. It is important to realise that Eq.(22) allows expressing $\mathbf{F}_{i}^{\mathrm{B}}$ in terms of the traction components associated with the two elements containing the node $i$; for two-node linear boundary elements such expression could be written as

$$
\mathbf{F}_{i}^{\mathrm{B}}=\sum_{k=i-1}^{i+1} \mathbf{M}_{k} \mathbf{t}_{k}
$$


where $\mathbf{t}_{k}$ collects the components of tractions of the node $k$. Once Eq.(23) is written for all the boundary element nodes belonging to $S$, the nodal forces $\mathbf{F}^{\mathrm{B}}$ appearing in the equilibrium equations, Eq.(18), can be expressed in terms of the boundary tractions $\mathbf{T}^{\mathrm{B}}$ appearing in Eq.(7) as

$$
\mathbf{F}^{\mathrm{B}}=\mathbf{M} \mathbf{T}^{\mathrm{B}}
$$

where $\mathbf{F}^{\mathrm{B}}, \mathbf{T}^{\mathrm{B}} \in \mathbb{R}^{2 m \times 1}$ and $\mathbf{M} \in \mathbb{R}^{2 m \times 2 m}$, with $m$ expressing the total number of boundary nodes/elements.

Exploiting Eq.(24), Eq.(7) can be written in a form to be used in conjunction with the VEM equations; in particular, remembering that $\mathbf{T}^{\mathrm{B}}=\mathbf{G}^{-1} \mathbf{H} \mathbf{U}^{\mathrm{B}}$, it is possible to write

$$
\mathbf{F}^{\mathrm{B}}=\mathbf{M T}^{\mathrm{B}}=\left(\mathbf{M G}^{-1} \mathbf{H}\right) \mathbf{U}^{\mathrm{B}}=\mathbf{K}^{\mathrm{B}} \mathbf{U}^{\mathrm{B}} .
$$

Considering the interface conditions in Eqs.(18), we can now combine the BEM equilibrium equations in Eq.25 with the VEM equilibrium equations Eq. 17 to obtain the final set of equations for the coupled system

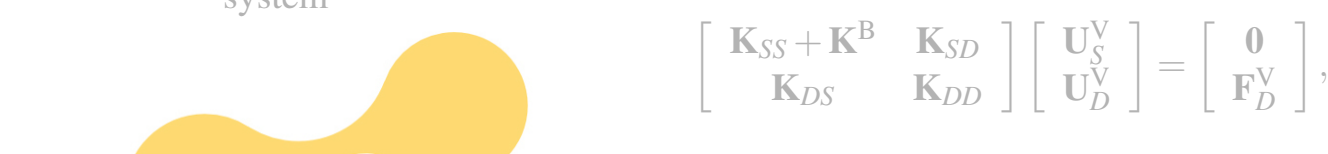

\section{NUMERICAL TESTS}

In this Section, we report a numerical application of the hybrid VEM/BEM formulation for the computational homogenisation problem of a composite material reinforced with fibres whose transversal section

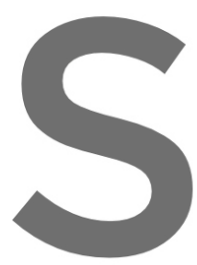
presents a more complex s

The problem consists of artificial digital sample

finite element discretisatio samples; (d) the computat
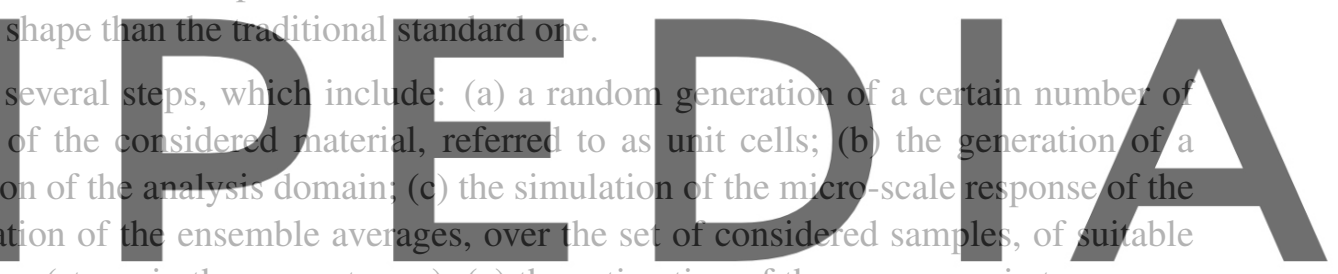

volume averaged quantities (stress in the present case); (e) the estimation of the macroscopic transverse

The present test's unit cells are generated by randomly scattering a given number of inclusions having

the same transversal section in a square domain. All the inclusions of a given sample present the same size but have a random orientation. The number of inclusions is determined by the parameter $\delta=\frac{L}{r}$, where $L$ is the unit cell's side length and $r$ is the radius of the circle that circumscribes the inclusion. Fig.(2) shows an example of a random generated microstructure with $\delta=35$.

The composite constituents are assumed to be isotropic in the analysis plane $x_{2}-x_{3}$, and their material constants are given in Table 1, in terms of transverse Young's modulus $E_{22}$ and transverse shear modulus $G_{23}$. Given the Poisson random distribution of fibres within the unit cell, the composite is considered isotropic at the macroscopic level, and its transverse behaviour can be entirely defined by two elastic modula: the plane strain bulk modulus $\bar{K}_{23}$ and the transverse shear modulus $\bar{G}_{23}$.

Sets of unit cells at increasing values of the parameter $\delta$ are considered, while the fibre volume fraction is kept constant at $V_{f}=0.25$. For each value of $\delta, N_{s}=50$ different random sample micromorphologies are generated. As shown in Fig.(3), a mesh of arbitrary polygonal virtual elements is used for the matrix subdomain and a mesh of linear boundary elements is adopted for the boundary of the inclusion subdomain. 

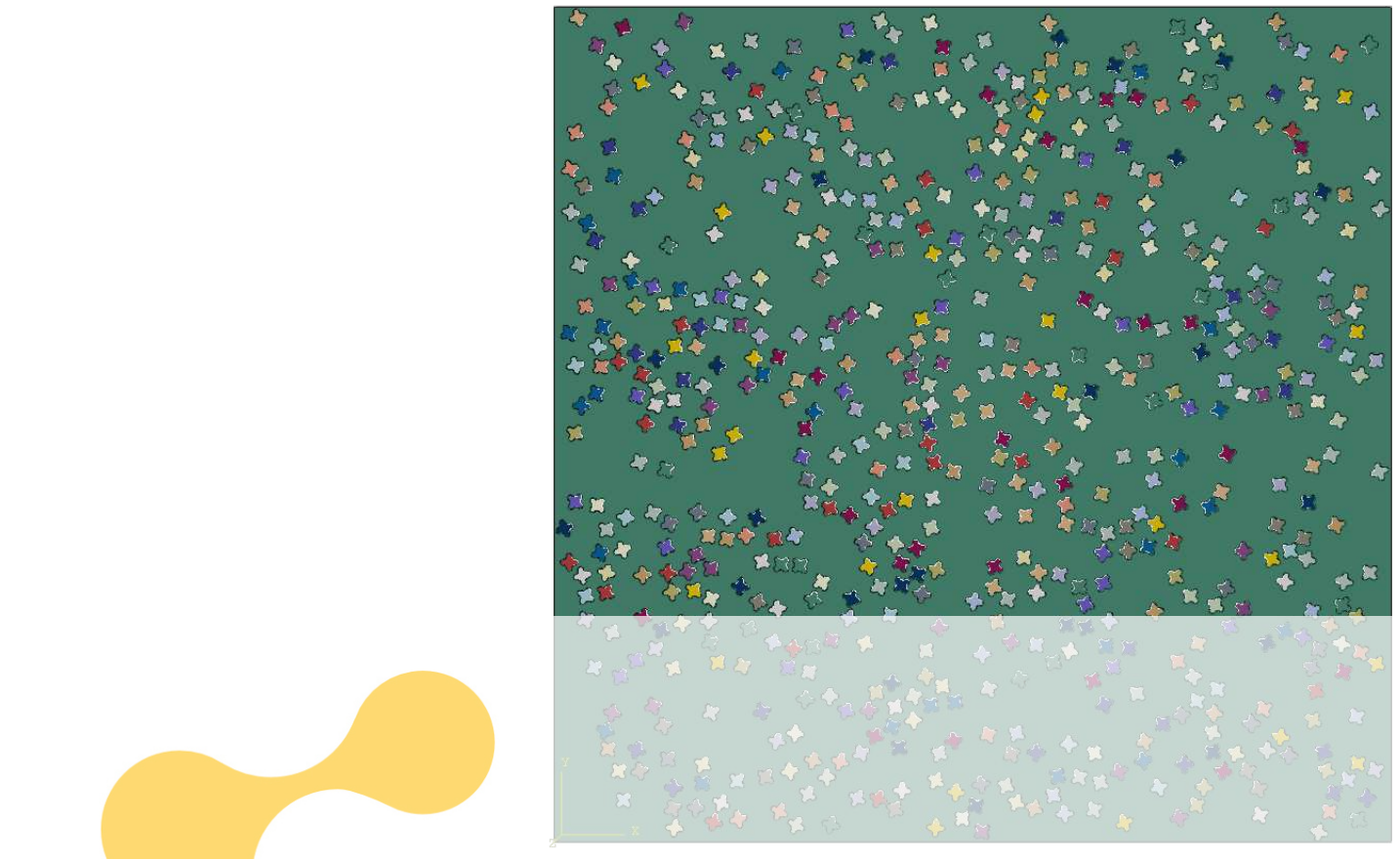

Figure 2: Example of a random generated geometry of a unit cells employed in the computational homogenization tests. $V_{f}=0.25$ and $\delta=35$
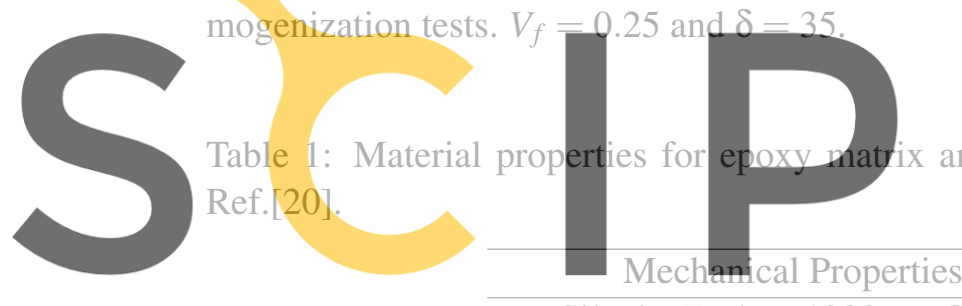

Silenka E-glass 1200 tex fibres
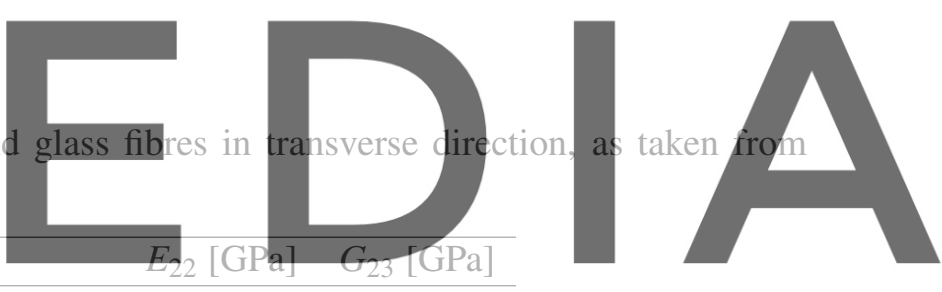

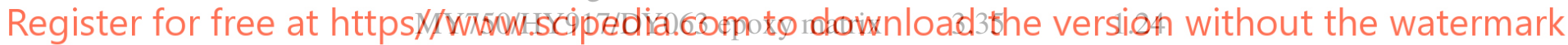

Each unit cell is subjected to three linearly independent sets of kinematic uniform boundary conditions, corresponding to three sets of enforced macro-strains whose general expression in Voigt notation is $\bar{\varepsilon}=\left\{\bar{\varepsilon}_{22}, \bar{\varepsilon}_{33}, 2 \bar{\varepsilon}_{23}\right\}$. The expressions for the three applied macro-strains are

$$
\bar{\varepsilon}^{1}=\{1,0,0\}, \quad \bar{\varepsilon}^{2}=\{0,1,0\}, \quad \bar{\varepsilon}^{3}=\{0,0,1\}
$$

The microstructural problem is solved under plane strain assumptions employing the proposed hybrid scheme, and the averaged stresses are then computed as volume averages of the local micro-stress tensor over the domain of the unit cells, as

$$
\overline{\boldsymbol{\sigma}}_{i j}=\frac{1}{\Omega} \int_{\Omega} \sigma_{i j}(\mathbf{x}) d \Omega=\frac{1}{\Omega}\left(\int_{\Omega^{V}} \sigma_{i j}(\mathbf{x}) d \Omega+\int_{\Omega^{B}} \sigma_{i j}(\mathbf{x}) d \Omega\right),
$$

where the domain integral is subdivided into contributions coming separately from the VE and BE regions. 

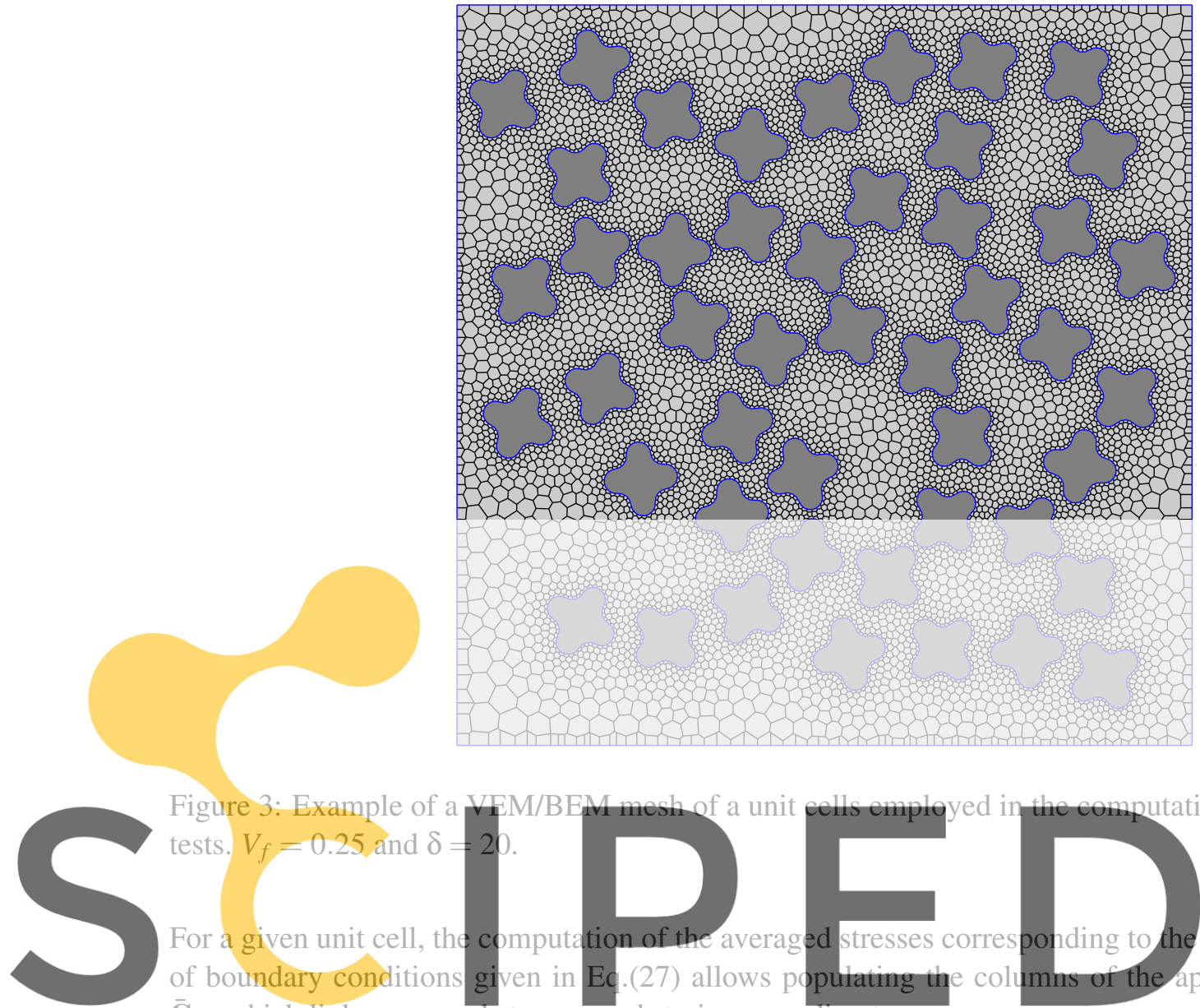

Figure 3: Example of a tests. $V_{f}=0.25$ and $\delta$

For a given unit cell, the of boundary conditions

$\overline{\mathbf{C}}_{m}$, which links averaged stresses and strains according to
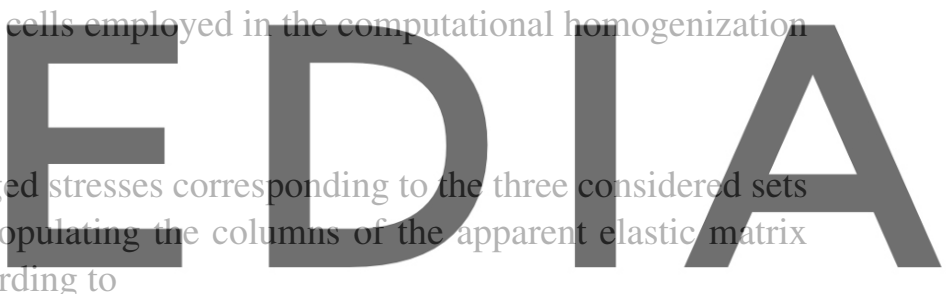

\section{Register for free at https//www.scipedia.comđe dodvnload the version without the watermark}

For each value of the parameter $\delta$, once the components of $\overline{\mathbf{C}}_{m}$ are computed for all the $N_{s}=50$ generated random unit cells, a macroscopic apparent constitutive matrix $\langle\overline{\mathbf{C}}\rangle$ is computed from the ensemble average of the components of $\overline{\mathbf{C}}_{m}$ over the $N_{s}$ samples, i.e.

$$
\langle\overline{\mathbf{C}}\rangle=\frac{1}{N_{s}} \sum_{m=1}^{N_{s}} \overline{\mathbf{C}}_{m}
$$

The apparent transverse elastic properties $\bar{K}_{23}$ and $\bar{G}_{23}$ associated to the considered value of $\delta$ are obtained from the ensemble averaged matrix $\langle\overline{\mathbf{C}}\rangle$.

Fig.(4) shows the computed values of $\bar{K}_{23}$ and $\bar{G}_{23}$ versus $\delta$, reporting both the values corresponding to individual samples and the ensemble-averaged values. In general, the scatter of the individual values decreases as the parameter $\delta$ increases. For $\delta=45$, the computed effective material properties are $\bar{K}_{23}=$ 5.89 and $\bar{G}_{23}=2.06$. These values fall within the analytical bounds predicted by the Hashin-Hill theory $[21,22]$ which are, for a volume fraction $V_{f}=0.25,5.72 \leq K_{23}^{H H} \leq 10.03$ and $1.86 \leq G_{23}^{H H} \leq 4.25$. 


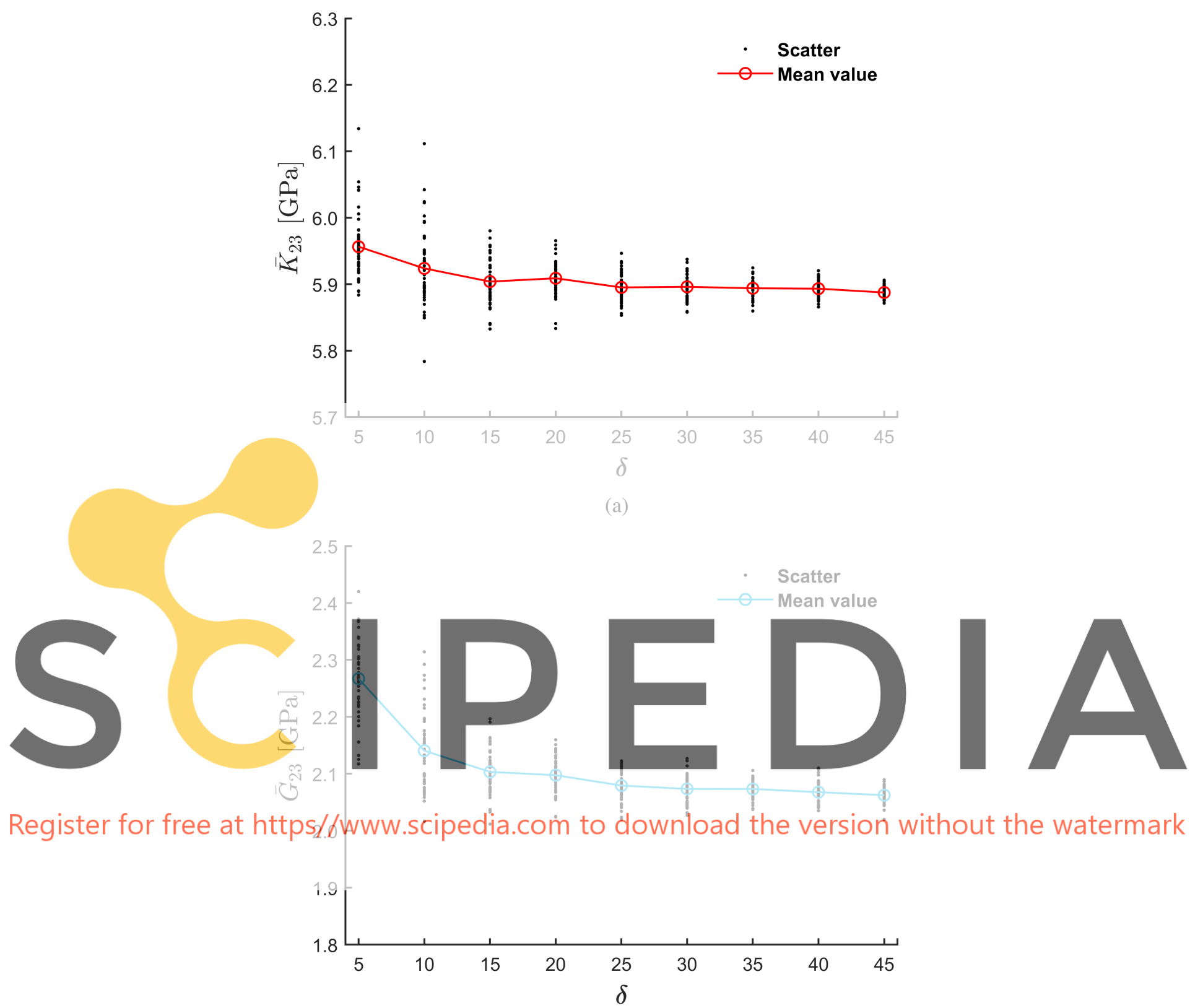

(b)

Figure 4: Apparent transverse elastic properties (a) $K_{23}$ and (b) $G_{23}$ as a function of the parameter $\delta$ as computed using the hybrid virtual-boundary element technique.

\section{CONCLUSIONS}

A hybrid technique coupling the Virtual and Boundary Element Methods for the computational homogenization of composite materials has been developed, implemented and tested by computing the transverse 
homogenised properties of a fibre-reinforced composite. It has been shown how the developed technique may be employed to by-pass problematic aspects potentially induced by the meshing of artificial microstructures with random geometrical features, which may become a particularly challenging task when the inclusions present involved shapes, and how the automatic mesh generation can be simplified and made more robust. In this framework, the inherent advantages of the formulation are two-fold: VEM's capability of providing accurate results with general polygonal mesh elements mitigates potential issues related to the quality of the mesh, avoiding the need of employing time-consuming regularisation techniques; modelling each inclusion with a single BEM super-element sensibly reduces the total number of degrees of freedom and, in turn, the overall cost of computational homogenization.

\section{ACKNOWLEDGEMENTS}

The authors acknowledge the support of the Italian Ministry of Education, University and Research MIUR - through the project DEVISU, funded under the scheme PRIN-2107 - Grant 22017ZX9X4K 006.

\section{REFERENCES}

[1] Zienkiewicz, O. C., Taylor, R. L., Nithiarasu, P., Zhu, J. Z.: The finite element method (Vol. 3). McGraw-hill, London (1977)

[2] Banerjee, P. K., Butterfield, R.: Boundary element methods in engineering science. McGraw-Hill, London (1981)

[3] Zienkiewicz, O. C., Kelly, D. W., Bettess, P.: The coupling of the finite element method and boundary solution procedures. International journal for numerical methods in engineering 11(2), 355375, (1977)

[4] Brebbia, C. A., Georgiou, P.: Combination of boundary and finite elements in elastostatics. Applied Mathematical Modelling 3(3), 212-220, (1979).

[5] Belytschko, T., Chang, H. S., Lu, Y. Y.: A variationally coupled finite element-boundary element method. Computers and structures 33(1), 17-20 (1989)

[6] Cruse, T. A., Osias, J. R.: Issues in merging the finite element and boundary integral equation methods. Mathematical and Computer Modelling 15(3-5), 103-118 (1991)

[7] Beirão da Veiga, L., Brezzi F., Cangiani, A., Manzini, G., Marini, L. D., Russo, A.: Basic principles of virtual element methods. Mathematical Models and Methods in Applied Sciences 23(01), 199214, (2013)

[8] Artioli, E., Marfia A., Sacco E.: High-order virtual element method for the homogenization of long fiber nonlinear composites. Computer Methods in Applied Mechanics and Engineering 341 571-585 (2018)

[9] Marino, M., Hudobivnik, B., Wriggers, P.: Computational homogenization of polycrystalline materials with the Virtual Element Method. Computer Methods in Applied Mechanics and Engineering 355, 349-372 (2019)

[10] Lo Cascio, M., Milazzo, A., Benedetti, I.: Virtual element method for computational homogenization of composite and heterogeneous materials. Composite Structures 232, 111523, (2020) 
[11] Lo Cascio, M., Benedetti, I. and Mantič, V.: Micro damage and cracking in fibre reinforced composites by a novel hybrid numerical technique. AIP Conference Proceedings 2309(1), 020001 (2020)

[12] Lo Cascio, M., Milazzo, A. and Benedetti, I.: Virtual Element Method: Micro-Mechanics Applications. Key Engineering Materials 827, 128-133 (2020)

[13] Benedetti, I., Aliabadi, M. H.: A three-dimensional grain boundary formulation for micro-structural modelling of polycrystalline materials. Computational Materials Science 67, 249-260, (2013)

[14] Gulizzi, V., Milazzo, A., Benedetti, I.: An enhanced grain-boundary framework for computational homogenization and micro-cracking simulations of polycrystalline materials. Computational Mechanics, 56(4), 631-651, (2015)

[15] Benedetti, I., Gulizzi, V., Mallardo, V.: A grain boundary formulation for crystal plasticity. International Journal of Plasticity, 83, 202-224, (2016)

[16] Benedetti, I., Gulizzi, V., Milazzo, A.: A microstructural model for homogenisation and cracking of piezoelectric polycrystals. Computer Methods in Applied Mechanics and Engineering, 357, 112595, (2019)

[17] Lo Cascio, M., Grifò, M., Milazzo, A., Benedetti, I.: Computational Homogenization of Heterogeneous Materials by a Novel Hybrid Numerical Scheme. Journal of Multiscale Modelling, 11(04), 2050008 (2020)

[18] Artioli, E., Beirão da Veiga, L., Lovadina, C., Sacco, E.: Arbitrary order 2D virtual elements for polygonal meshes: part I, elastic problem. Computational Mechanics 60(3), 355-377 (2017)

[19] Beirão da Veiga, L., Brezzi, F., Marini, L. D.: Virtual elements for linear elasticity problems. SIAM Journal on Numerical Analysis 51(2), 794-812 (2013)

[20] Soden, P. D., Hinton, M. J., Kaddour, A. S.: Lamina properties, lay-up configurations and loading conditions for a range of fibre reinforced composite laminates. Failure criteria in fibre-reinforcedpolymer composites. Elsevier, (2004)

[21] Hill, R.: Elastic properties of reinforced solids: some theoretical principles. Journal of the Mechanics and Physics of Solids, 11(5), 357-372 (1963)

[22] Hashin, Z.: On elastic behaviour of fibre reinforced materials of arbitrary transverse phase geometry. Journal of the Mechanics and Physics of Solids, 13(3), 119-134 (1965) 